\title{
De compras para la salud: Sodio ${ }^{1}$
}

\author{
Wendy J. Dahl y Lauren Foster ${ }^{2}$
}

\section{¿Por qué necesito el sodio?}

El sodio es un mineral que se encuentra en la sal de mesa. Es necesario para mantener un balance de fluidos y el volumen de sangre. El consumo excesivo de sodio puede causar presión alta en la sangre e incrementar el riesgo de enfermedades del corazón y derrames cerebrales.

\begin{tabular}{|c|c|c|}
\hline \multicolumn{3}{|l|}{ NUTRITION FACTS } \\
\hline \multicolumn{3}{|c|}{$\begin{array}{l}\text { Serving size: } 1 / 2 \text { cup }(123 g) \text { pumpkin, low- } \\
\text { sodium, canned }\end{array}$} \\
\hline \multicolumn{3}{|l|}{ Amount Per Serving } \\
\hline Calories $\quad 40$ & Calories from Fat & 5 \\
\hline \multicolumn{3}{|c|}{$\%$ Daily Value* } \\
\hline Total Fat $\mathrm{Og}$ & & $0 \%$ \\
\hline Saturated Fat $0 \mathrm{~g}$ & & $0 \%$ \\
\hline \multicolumn{3}{|l|}{ Trans Fat $0 \mathrm{~g}$} \\
\hline Cholesterol Omg & & $0 \%$ \\
\hline Sodium $5 \mathrm{mg}$ & & $0 \%$ \\
\hline Total Carbohydrate $10 \mathrm{~g}$ & & $3 \%$ \\
\hline Dietary Fiber $4 \mathrm{~g}$ & & $16 \%$ \\
\hline \multicolumn{3}{|l|}{ Sugars $4 \mathrm{~g}$} \\
\hline \multicolumn{3}{|l|}{ Protein $1 \mathrm{~g}$} \\
\hline Vitamin A & Vitamin C & $8 \%$ \\
\hline Calcium & Iron & $10 \%$ \\
\hline
\end{tabular}

\section{¿Cuánto sodio necesito?}

Para una salud óptima, los expertos recomiendan que los adultos no se excedan de $2300 \mathrm{mg}$ de sodio, lo cual equivale a más o menos una cucharadita de sal al día (1). Los adultos jóvenes necesitan $1500 \mathrm{mg}$ de sodio por día mientras que adultos mayores de 70 años necesitan solo 1200 mg por día. La mayoría de personas consumen mucho más del sodio que necesitan.

Los individuos con alta presión arterial u otra condición de la salud pueden ser puestos en una restricción de sodio por su médico. La mayoría de las dietas bajas en sodio tienen un consumo limitado de 1500 mg por día (1).

\section{¿Qué alimentos son altos es sodio?} Alimentos procesados

En los Estados Unidos, la mayor fuente de sodio en la dieta son los alimentos preparados y procesados tales como los alimentos enlatados, las carnes embutidas y las comidas congeladas. Frecuentemente se añade sal para preservar el alimento y añadir sabor (2). Por ejemplo, un pedazo de pizza congelado tiene cerca de $900 \mathrm{mg}$ de sodio. Las comidas de caja con condimentos pre-empacados son usualmente también altos en sodio. Una sola porción de hamburguesa con queso o macarrones de Hamburguer Helper ${ }^{\circledast}$ tiene 914 mg de sodio. Podemos disminuir nuestro consumo diario de sodio si evitamos las comidas procesadas.

1. The English version of this document is FCS10-06/FS154 Shopping for Health: Sodium. Este documento, FSHN10-06s, es uno de una serie de publicaciones del Food Science and Human Nutrition Department, Servicio de Extensión Cooperativa de la Florida, Instituto de Alimentos y Ciencias Agrícolas, Universidad de la Florida (UF/IFAS). Fecha de primera publicación marzo 2013. Visite el sitio web en EDIS http://edis.ifas.ufl.edu/.

2. Wendy J. Dahl, PhD, profesora asistente, y Lauren Foster, BS, Food Science and Human Nutrition Department, University of Florida, Gainesville, FL 32611. 


\section{Condimentos altos en sodio}

Los condimentos tales como los aderezos para la ensalada o la salsa de soya pueden incrementar significativamente el contenido de sodio en las comidas. Una cucharada de salsa de soya tiene cerca de $1000 \mathrm{mg}$ de sodio (2), y una cucharada de aderezo de ensalada regular tiene cerca de $200 \mathrm{mg}$.

\section{De compras para alimentos bajos}

\section{en sodio}

Una dieta baja en sodio no requiere que usted deje de consumir ciertos grupos de alimentos, pero si requiere que usted sea un comprador más consciente. Las diferentes marcas de los mismos alimentos pueden tener dramáticamente diferentes contenidos de sodio, así que es importante que siempre lea las etiquetas de los alimentos. De acuerdo con la FDA (Administración de drogas y alimentos), los alimentos bajos en sodio contienen $140 \mathrm{mg}$ o menos por porción (3).

\section{Vegetales}

Cuando esté comprando vegetales, considere como estos han sido procesados. Los vegetales enlatados frecuentemente contienen altos niveles de sodio que ha sido añadido para mejorar el sabor y la vida útil. Si escoge vegetales frescos o congelados, en vez de vegetales enlatados va a reducir su consumo diario de sodio siempre que usted no añada sal de mesa cuando los esté cocinando. La tabla 1 tiene listados varios vegetales y su contenido de sodio basado en como han sido procesados (4).

Si añadimos salsas o aderezos altos en sodio cuando preparamos los vegetales podemos afectar significativamente el contenido del sodio.

\section{Pan}

Aunque no siempre los panes son "salados," estos pueden ser altos en sodio. En la tabla 2 tenemos una lista de los diferentes tipos de pan y la cantidad de sodio en cada uno (4). Las alternativas de pan bajas en sodio pueden ser tortillas de maíz o galletas sin sal.

\section{Carne}

Así como con los vegetales, las carnes también varían en el contenido del sodio dependiendo de su preparación. Las carnes procesadas tales como las carnes embutidas, ahumadas o curadas son altas en sodio. La tabla 3 compara la cantidad de sodio en la carne asada fresca y la de la carne embutida procesada (4).
La porción recomendada para la mayoría de carnes embutidas es 2 onzas. Sin embargo, muchos sándwiches de restaurante tipo deli proveen cerca de 5-6 onzas de carne. Una porción de salami de 2 onzas provee casi una tercera parte de la cantidad de sodio recomendada. ¡Si usted prepara un sándwich con dos tajadas de pan blanco y 2 onzas de carne embutida, ya esta consumiendo cerca de la mitad del consumo adecuado de sodio!

\section{Cereal}

Los cereales de desayuno que vienen listos para comer varían en gran medida en sus niveles de sodio. Mientras hay algunos cereales bajos en sodio disponibles, la mayoría tiene altos niveles de sal añadida. Los contenidos de sodio varían desde menos de $10 \mathrm{mg}$ por porción en trigo triturado hasta más de 350 mg por porción en Kellogs de salvado con pasas. En la tabla 4 están algunos cereales bajos en sodio.

\section{Queso}

Los quesos procesados son generalmente altos en sal y deben ser eliminados en las dietas bajas en sodio. Algunos supermercados venden marcas bajas en sodio, pero estas pueden ser difíciles de encontrar. En la tabla 5 está el contenido de sodio de varios quesos.

\section{La merienda}

Muchas meriendas populares, tales como las papitas fritas y las galletas son altas en sales añadidas. Los pretzels o galletas saladas tienen cerca de $500 \mathrm{mg}$ de sodio por porción y las palomitas de maíz tienen cerca de $350 \mathrm{mg}$. $\mathrm{Al}$ igual que otros grupos de alimentos, algunas meriendas pueden no tener un sabor salado, pero aún así ser altas en sodio. Por ejemplo, un pudín de Jello ${ }^{\circledast}$ de tamaño de una porción tiene casi $200 \mathrm{mg}$ de sodio.

Para satisfacer la necesidad de merendar sin la sal adicional, usted puede ensayar con pasas, frutas secas, o maní sin sal como alternativas. Otra opción puede ser buscar marcas que ofrezcan una versión de estas meriendas sin sal. FritoLay ofrece una línea de papitas fritas bajas en sal llamadas Pinch of Salt ${ }^{\oplus}$ chips, y Snyder's of Hanover vende pretzels miniatura que no contienen sal. Estas dos opciones tienen solo $75 \mathrm{mg}$ de sodio por porción.

\section{¡Sea un comprador informado!}

Asegúrese siempre de leer los etiquetas de los alimentos para chequear el contenido de sodio. La cantidad de sodio por porción está listada usualmente en mg debajo de los nutrientes, como se muestra abajo. También está listado 
el porcentaje del valor diario, el cual está basado en un consumo diario de $2000 \mathrm{mg}$ de sodio.

La próxima vez que esté yendo de compras, tenga en mente las siguientes guías para limitar el consumo de sodio.

- Escoja vegetales frescos o congelados en vez de los enlatados.

- Escoja carnes frescas o congeladas en vez de carnes que han sido ahumadas, curadas, enlatadas o secas.

- Escoja aderezos de ensaladas bajos en sodio en vez de aderezos regulares.

- Escoja sopas con sodio reducido o prepare las sopas en casa.

- Evite las mezclas preempacadas de Biscuits y waffle. En cambio, escoja cereales bajos en sodio o avena.

- Sustituya condimentos altos en sodio por hierbas frescas.

- Sustituya meriendas altas en sodio y panes por galletas sin sal y tortillas de maíz.

\section{Referencias}

1. Mayo Foundation for Medical Education and Research. 2008. Sodium: Are you getting too much? Retrieved December 16, 2009 from http://www.mayoclinic.com/ health/sodium/NU00284

2. U.S. Department of Health and Human Services and U.S. Department of Agriculture. Dietary Guidelines for Americans, 2005. 6th Edition, Washington, DC: U.S. Government Printing Office, January 2005.

3. U.S. Department of Health and Human Services. 2004. Appendix A: Definitions of Nutrient Content Claims. In Guidance for industry:A food labeling guide (Appendix A). Retrieved December 14, 2009 from http://www.fda. gov/Food/GuidanceComplianceRegulatoryInformation/ GuidanceDocuments/FoodLabelingNutrition/FoodLabelingGuide/ucm064911.htm

4. U.S. Department of Agriculture, Agricultural Research Service. 2009. National Nutrient Database for Standard Reference, Release 22. Nutrient Data Laboratory Home Page, http://www.ars.usda.gov/ba/bhnrc/ndl

Tabla 1. Contenido de sodio en los vegetales

\begin{tabular}{|c|c|c|c|c|}
\hline & \multicolumn{4}{|c|}{ Sodio (mg por una porción de 1/2 taza) } \\
\hline & Frescos & Congelados (sin sal) & Enlatados & Enlatados (bajo o no sodio) \\
\hline Espárragos & 1 & 3 & 346 & 29 \\
\hline Zanahoria & 42 & 43 & 490 & 240 \\
\hline Maíz Amarillo & 4 & 43 & 286 & 15 \\
\hline Judías verdes & 11 & 3 & 311 & 17 \\
\hline Guisantes & 3 & 58 & 214 & 11 \\
\hline Espinacas & 3 & 4 & 373 & 88 \\
\hline
\end{tabular}

Tabla 2. Contenido de sodio típico en el pan

\begin{tabular}{|c|c|}
\hline Pan & Sodio (mg/tajada) \\
\hline Italiano & 117 \\
\hline Granos Mixtos & 109 \\
\hline Pita, Blanca (4") & 150 \\
\hline Pan Integral de Centeno & 174 \\
\hline Pasas & 81 \\
\hline Centeno & 211 \\
\hline Blanco & 128 \\
\hline Integral & 132 \\
\hline
\end{tabular}


Copia de archivo: para las recomendaciones actuales, consulte http://edis.ifas.ufl.edu o su oficina de extensión local.

Tabla 3. Contenido de sodio en carne asada y carne embutida

\begin{tabular}{|c|c|c|}
\hline & \multicolumn{2}{|c|}{ Sodio (mg por una porción de 2 onzas) } \\
\hline Pollo & Carne asada & Carne embutida \\
\hline Pavo & 43 & 705 \\
\hline Carne de res & 40 & 705 \\
\hline
\end{tabular}

Tabla 4. Cereales bajos en sodio (<140 mg)

\begin{tabular}{|c|c|}
\hline Cereal & Sodio (mg/porción) \\
\hline Cereales de germen de trigo de arroz inflado, trigo inflado y tostado & 0 \\
\hline Kellogg's Frosted Mini-Wheats ${ }^{\circledast}$ & 5 \\
\hline Kellogg's Mini-Wheats Original ${ }^{\circledast}$ & 0 \\
\hline Quaker $^{\circledast} 100 \%$ Cereal natural con avena, miel y pasas & 127 \\
\hline Kellogg's Smacks ${ }^{\circledast}$ & 51 \\
\hline Kellogg's All-Bran Original ${ }^{\circledR}$ & 81 \\
\hline Granola con pasas bajo en grasa Quaker ${ }^{\oplus}$ & 144 \\
\hline Kellogg's Corn Pops ${ }^{\circledR}$ & 124 \\
\hline \multicolumn{2}{|l|}{$\mathrm{mg}=$ miligramos } \\
\hline
\end{tabular}

Tabla 5. El contenido de sodio en los quesos típicos

\begin{tabular}{|c|c|}
\hline Queso & Sodio (mg/oz) \\
\hline Parmesano & 433 \\
\hline Americano & 422 \\
\hline Azul & 395 \\
\hline Suizo & 54 \\
\hline Provolone & 248 \\
\hline Mozzarella & 178 \\
\hline Cheddar & 176 \\
\hline
\end{tabular}

